\title{
Nitrogen defects and ferromagnetism of Cr-doped AlN diluted magnetic semiconductor from first principles
}

\author{
Li-Jie Shi $*$ Li-Fang Zhu, Yong-Hong Zhao, and Bang-Gui Liv \\ Institute of Physics, Chinese Academy of Sciences, Beijing 100190, China \\ Beijing National Laboratory for Condensed Matter Physics, Beijing 100190, China
}

(Dated: November 3, 2018)

\begin{abstract}
High Curie temperature of $900 \mathrm{~K}$ has been reported in Cr-doped AlN diluted magnetic semiconductors prepared by various methods, which is exciting for spintronic applications. It is believed that $\mathrm{N}$ defects play important roles in achieving the high temperature ferromagnetism in good samples. Motivated by these experimental advances, we use a full-potential density-functional-theory method and supercell approach to investigate $\mathrm{N}$ defects and their effects on ferromagnetism of $(\mathrm{Al}, \mathrm{Cr}) \mathrm{N}$ with $\mathrm{N}$ vacancies $\left(V_{\mathrm{N}}\right)$. We investigate the structural and electronic properties of the $V_{\mathrm{N}}$, single $\mathrm{Cr}$ atom, $\mathrm{Cr}-\mathrm{Cr}$ atom pairs, $\mathrm{Cr}-V_{\mathrm{N}}$ pairs, and so on. In each case, the most stable structure is obtained by comparing different atomic configurations optimized in terms of the total energy and the force on every atom, and then is used to calculate the defect formation energy and study the electronic structures. Our total energy calculations show that the nearest substitutional Cr-Cr pair with the two spins in parallel is the most favorable and the nearest $\mathrm{Cr}-V_{\mathrm{N}}$ pair makes a stable complex. Our formation energies indicate that $V_{\mathrm{N}}$ regions can be formed spontaneously under N-poor condition because the minimal $V_{\mathrm{N}}$ formation energy equals $-0.23 \mathrm{eV}$ or $\mathrm{Cr}$-doped regions with high enough concentrations can be formed under N-rich condition because the $\mathrm{Cr}$ formation energy equals 0.04 $\mathrm{eV}$, and hence real Cr-doped AlN samples are formed by forming some Cr-doped regions and separated $V_{\mathrm{N}}$ regions and through subsequent atomic relaxation during annealing. Both of the single $\mathrm{Cr}$ atom and the $\mathrm{N}$ vacancy create filled electronic states in the semiconductor gap of AlN. N-vacancies enhance the ferromagnetism by adding $1 \mu_{B}$ to the Cr moment each, but reduce the ferromagnetic exchange constants between the spins in the nearest $\mathrm{Cr}-\mathrm{Cr}$ pairs. These calculated results are in agreement with experimental observations and facts of real Cr-doped AlN samples and their synthesis. Our first-principles results are useful to elucidating the mechanism for the ferromagnetism and exploring high-performance $\mathrm{Cr}$-doped AlN diluted magnetic semiconductors.
\end{abstract}

\section{INTRODUCTION}

The diluted magnetic semiconductor (DMS) is of great interest because it has merits of both semiconductor and spin magnetism. Especially the possibility of hightemperature ferromagnetism in DMSs has inspired scientists from all the world to explore new promising DMS materials and study their physical properties for spintronic applications 1.2 . (Ga,Mn)As has been studied substantially because of its close relation with important GaAs semiconductor, and its ferromagnetism is believed to be induced by hole carriers ${ }^{3.4}$. Generally speaking, it is believed that at least $500 \mathrm{~K}$ as Curie temperature is necessary for real spintronic devices to work at room-temperature ${ }^{5}$. Curie temperature higher than $500 \mathrm{~K}$ is achieved in various transitionmetal doped oxides $6,7,8,9,10,11,12,13,14$ and nitrides 15,16 . For $\mathrm{Cr}$ doped $\mathrm{AlN}$, or $(\mathrm{Al}, \mathrm{Cr}) \mathrm{N}$, high Curie temperature of $900 \mathrm{~K}$ has been reported by several groups 17,18,19. It is shown that the high temperature ferromagnetism in $(\mathrm{Al}, \mathrm{Cr}) \mathrm{N}$ materials is intrinsic, not produced by secondary phases $16,17,18,19,20,21,22$. Naturally, such high Curie temperatures make the materials promising for practical spintronic applications $\frac{1}{*}$. Although ferromagnetism of $(\mathrm{Ga}, \mathrm{Mn})$ As DMS materials is believed to be induced by their effective carriers ${ }^{3.4}$, the high temperature ferromagnetism of $(\mathrm{Al}, \mathrm{Cr}) \mathrm{N}$ materials must be caused by different mechanism because some single-phase $(\mathrm{Al}, \mathrm{Cr}) \mathrm{N}$ samples with high Curie temperatures are highly insulating 21,22 (the same as pure $\mathrm{AlN}^{23}$ ) or in the regime of variable-range hopping conduction $\underline{\underline{ }}$ (similar to $\left.(\mathrm{Ga}, \mathrm{Cr}) \mathrm{N}^{25}\right)$. The ferromagnetism should be attributed to $p d$ hybridization of $\mathrm{Cr} d$ states and $\mathrm{N} p$ ones $26,27,28$. On the other hand, defects and impurities are believed to play important roles in DMS materials of wide semiconductor gaps $14,21,22,29,30,31,32,33$. Nitrogen pressure during synthesis is shown to affect the ferromagnetism in resultant samples $21,22,30$. It appears that reduced $\mathrm{N}$ content enhances the ferromagnetism, being in contrast with first-principles-calculated trend of $\mathrm{N}$-vacancies in the case of GaN-based DMSs ${ }^{34}$. Therefore, it is highly desirable to investigate effect of $\mathrm{N}$ content on the high temperature ferromagnetism in $(\mathrm{Al}, \mathrm{Cr}) \mathrm{N}$ materials, and in return its elucidation is helpful to obtain deeper insight into the essential mechanism of the high temperature ferromagnetism.

In this paper we use a full-potential density-functionaltheory method and $3 \times 3 \times 2$ supercell approach to investigate $\mathrm{N}$ defects and their effects on ferromagnetism of $(\mathrm{Al}, \mathrm{Cr}) \mathrm{N}$ with $\mathrm{N}$ vacancies $\left(V_{\mathrm{N}}\right)$, namely $\mathrm{Cr}_{x} \mathrm{Al}_{1-x} \mathrm{~N}_{1-y}$. We obtain the most stable structures of $\mathrm{Al}_{36} \mathrm{~N}_{35}$ (one $V_{\mathrm{N}}$ in the supercell), $\mathrm{CrAl}_{35} \mathrm{~N}_{36}$ (one substitutional $\mathrm{Cr}$ in the supercell), $\mathrm{CrAl}_{35} \mathrm{~N}_{35}$ (one $V_{\mathrm{N}}$ and one substitutional $\mathrm{Cr}$ atom in the supercell), $\mathrm{Cr}_{2} \mathrm{Al}_{34} \mathrm{~N}_{36}$ (two substitutional $\mathrm{Cr}$ atoms in the supercell), and so on by comparing their different atomic configurations optimized in terms of the total energy and the force on every atom, and thereby calculate their defect formation energies. Then we in- 
vestigate the structural and electronic properties of the $V_{\mathrm{N}}$, single $\mathrm{Cr}$ atom, $\mathrm{Cr}-\mathrm{Cr}$ pairs, and $\mathrm{Cr}-V_{\mathrm{N}}$ pairs. Without $\mathrm{N}$ vacancy, the nearest substitutional $\mathrm{Cr}$-Cr pair with their moments orienting in parallel is the most favorable in total energy. The nearest pair of an $\mathrm{N}$ vacancy and a single $\mathrm{Cr}$ atom forms a stable complex of $\mathrm{Cr}^{-}$and $V_{\mathrm{N}}^{+}$. Our calculated formation energies indicate that $V_{\mathrm{N}}$ regions can be formed spontaneously under N-poor condition or high enough $\mathrm{Cr}$ concentrations can be formed under N-rich condition, and hence real $\mathrm{Al}_{1-x} \mathrm{Cr}_{x} \mathrm{~N}_{1-y}$ samples are formed by forming Cr-doped regions and some separated $V_{\mathrm{N}}$ regions and through subsequent atomic relaxation during annealing. The doped $\mathrm{Cr}$ atom and the $\mathrm{N}$-vacancy create filled electronic states in the semiconductor gap of AlN. $V_{\mathrm{N}}$ enhances the ferromagnetism by giving one electron to a $\mathrm{Cr}$ ion to add $1 \mu_{B}$ to the $\mathrm{Cr}$ moment, which is in agreement with experimental observation that low $\mathrm{N}$ pressure during synthesis is helpful to obtaining better ferromagnetic $\mathrm{Al}_{1-x} \mathrm{Cr}_{x} \mathrm{~N}_{1-y}$ samples 22.30 . On the other hand, $V_{\mathrm{N}}$ reduces the ferromagnetic exchange constants between the spins in the nearest $\mathrm{Cr}$ Cr pairs, which is consistent with experimental fact that too many $\mathrm{N}$ vacancies are harmful to the ferromagnetism when the synthesis temperature is high enough to allow doped $\mathrm{Cr}$ atoms to diffuse. These first-principles results are useful to exploring high-performance $(\mathrm{Al}, \mathrm{Cr}) \mathrm{N}$ DMS samples and understanding the high temperature ferromagnetism.

The remaining part of this paper is organized as follows. We shall present our computational method and parameters in next section. In the third section, we shall present our main results of optimized magnetic structures (through geometric and internal atomic position optimization) and defect formation energies. In the fourth section, we shall present main electronic structures, including energy bands and electron density distributions. In the fifth section, we shall discuss the ferromagnetism correlated with $\mathrm{N}$ defects in real samples. Finally, we shall give our main conclusion in the sixth section.

\section{COMPUTATIONAL METHOD AND PARAMETERS}

We use a first-principles method and supercell approach to study wurtzite AlN with doped $\mathrm{Cr}$ atoms and $\mathrm{N}$ defects (including nitrogen vacancies and nitrogen interstitials). We use a $3 \times 3 \times 2$ supercell as showed in Fig. 1 and substitute a $\mathrm{Cr}$ atom for an $\mathrm{Al}$ atom to model $\mathrm{Cr}$ doping concentration $2.78 \%$ realized in MBE samples $16,18,19,21$. This supercell is not very large to simulate real experimental conditions, but it is large enough to model the representative concentrations of both doped $\mathrm{Cr}$ and $V_{\mathrm{N}}$ and main properties of $\mathrm{Cr}-\mathrm{Cr}$ and $\mathrm{Cr}-V_{\mathrm{N}}$ interactions, especially considering that our calculations are done with full-potential linear augmented plane wave (FLAPW) method within the density-functional theory $(\mathrm{DFT})^{35}$. Using such supercell is also supported by the

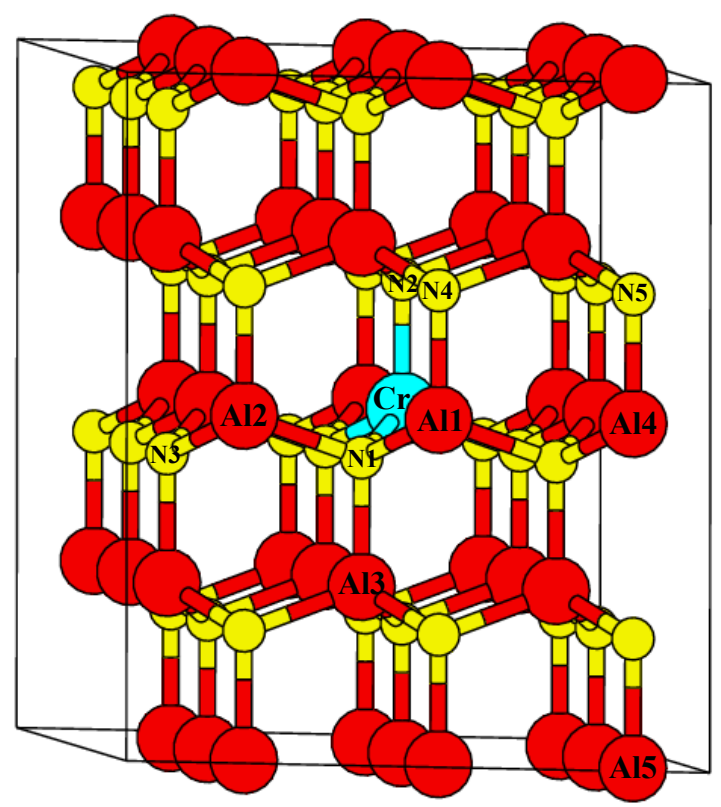

FIG. 1: (color online). Schematic structure of Cr-substituted $3 \times 3 \times 2$ wurtzite AlN supercell, $\mathrm{CrAl}_{35} \mathrm{~N}_{36}$. The centering largest (cyan or white) ball is the first $\mathrm{Cr}$ atom, the smallest (yellow or light gray) ones are $\mathrm{N}$ atoms, and the other (red or gray) ones are $\mathrm{Al}$ atoms. An $\mathrm{N}$ vacancy can be created by removing an $\mathrm{N}$ atom, and the second substitutional $\mathrm{Cr}$ atom can be introduced by substituting a $\mathrm{Cr}$ for an $\mathrm{Al}$ atom. For examples, $V_{\mathrm{N} 1}$ (or $V_{1}$ ) is made by removing N1, and $\mathrm{Cr} 3$ by substituting a $\mathrm{Cr}$ atom for $\mathrm{Al} 3$.

following calculated results that the total energy of both $\mathrm{Cr}_{\mathrm{Al}}-\mathrm{Cr}_{\mathrm{Al}}$ and $\mathrm{Cr}_{\mathrm{Al}}-V_{\mathrm{N}}$ pairs substantially increases with increasing the $\mathrm{Cr}_{\mathrm{Al}}-\mathrm{Cr}_{\mathrm{Al}}$ and $\mathrm{Cr}_{\mathrm{Al}}-V_{\mathrm{N}}$ distances, respectively. The supercell consists of one $\mathrm{Cr}$ atom, $35 \mathrm{Al}$ atoms, and $36 \mathrm{~N}$ atoms and its space group is No. 156 . Such a supercell for the pure AlN consists of $36 \mathrm{Al}$ and 36 $\mathrm{N}$ atoms. The second $\mathrm{Cr}$ atom is introduced by substituting for one of $\mathrm{Al}$ atoms in the supercell. For examples, $\mathrm{Cr} 1$ is made by substituting a $\mathrm{Cr}$ atom for the $\mathrm{Al}$ atom labelled as Al1 in Fig. 1. A nitrogen vacancy $V_{\mathrm{N}}$ is created by removing one $\mathrm{N}$ atom. For examples, $V_{\mathrm{N} 1}$ (or $V 1$ ) is made by removing the $\mathrm{N}$ atom labelled as N1. An interstitial $\mathrm{N}$ atom is introduced by adding an $\mathrm{N}$ atom in the supercell. We obtain an octahedral $\mathrm{N}$ interstitial $\mathrm{N}_{\mathrm{T}}$ if adding an $\mathrm{N}$ atom at an octahedral site, or obtain tetrahedral $\mathrm{N}$ interstitial $\mathrm{N}_{\mathrm{T}}$ if adding an $\mathrm{N}$ atom at a tetrahedral site. We optimize lattice constants to minimize the total energy, and at the same time optimize internal atomic coordinates to make the force on every atom less than $0.04 \mathrm{eV} / \AA$. All the electronic structures and magnetic moments are calculated with equilibrium lattice constants.

All the presented results are calculated with the fullpotential linear augmented plane wave method within the density-functional theory $\stackrel{35}{ }$, as implemented in the package WIEN2 $\mathrm{k}^{36}$, although we also use pseudo potential method to do some preliminary structural optimizations. 
The generalized gradient approximation (GGA) ${ }^{37}$ is used as the exchange-correlation potential for all our presented results, and some important cases are confirmed by localdensity-approximate calculations ${ }^{38}$. Full relativistic calculations are done for core states and the scalar approximation is used for the others, with the spin-orbit coupling being neglected ${ }^{39}$. The radii of muffin-tin spheres, $R_{\mathrm{mt}}$, are adjusted so as to achieve the best accuracy of self-consistent calculations. We use the parameters $R_{\mathrm{mt}} \cdot K_{\max }=8.0$ and make the sphere harmonic expansion upto $l_{\max }=10$ in the spheres. The Brillouin zone integrations are performed with the special k-point method over a $5 \times 5 \times 3$ Monkhorst-Pack mesh. A convergence standard, $\int\left|\rho_{n}-\rho_{n-1}\right| d r<0.00005\left(\rho_{n}\right.$ and $\rho_{n-1}$ are output and input charge density, respectively), is used for all our self-consistent calculations.

\section{OPTIMIZED STRUCTURES AND DEFECT FORMATION ENERGIES}

We optimize fully the lattice structures and internal atomic positions for $\mathrm{N}$ vacancy $\left(\mathrm{Al}_{36} \mathrm{~N}_{36}+V_{\mathrm{N}}=\mathrm{Al}_{36} \mathrm{~N}_{35}\right)$, $\mathrm{Cr}$ doped AlN $\left(\mathrm{CrAl}_{35} \mathrm{~N}_{36}\right)$, Cr-atom plus $\mathrm{N}$-vacancy $\left(\mathrm{CrAl}_{35} \mathrm{~N}_{36}+V_{\mathrm{N}}=\mathrm{CrAl}_{35} \mathrm{~N}_{35}\right)$, Cr-atom plus tetrahedral $\mathrm{N}$ interstitial $\left(\mathrm{CrAl}_{35} \mathrm{~N}_{36}+\mathrm{N}_{\mathrm{T}}\right)$, and Cr-atom plus octahedral $\mathrm{N}$ interstitial $\left(\mathrm{CrAl}_{35} \mathrm{~N}_{36}+\mathrm{N}_{\mathrm{O}}\right)$. We can have various atomic configurations for pairs of $\mathrm{Cr}-V_{\mathrm{N}}, \mathrm{Cr}-\mathrm{N}_{\mathrm{T}}$, and $\mathrm{Cr}-\mathrm{N}_{\mathrm{O}}$ with given distance, and they correspond to different total energies. As for $\mathrm{CrAl}_{35} \mathrm{~N}_{36}+V_{\mathrm{N}}$, the total energy changes with the distance between $\mathrm{Cr}$ and $V_{\mathrm{N}}$, and the lowest total energy is reached when $\mathrm{Cr}$ and $V_{\mathrm{N}}$ are the nearest neighbors $\mathrm{Cr}-\mathrm{V} 1$ (connected with an approximately horizontal $\mathrm{Cr}-\mathrm{N}$ bond). The next lowest total energy $(0.23 \mathrm{eV})$ is reached by $\mathrm{Cr}-V 2$. For double $\mathrm{Cr}$ doped system, the total energy depends on the $\mathrm{Cr}-\mathrm{Cr}$ distance, and the lowest total energy is reached when the two $\mathrm{Cr}(\mathrm{Cr}$ and $\mathrm{Cr} 1)$ are in the same layer and connected with the same $\mathrm{N}$ (consistent with previous result ${ }^{40}$ ). The next lowest total energy $(0.28 \mathrm{eV})$ is reached by $\mathrm{Cr}-\mathrm{Cr} 3$ and other equivalent $\mathrm{Cr}-\mathrm{Cr}$ pairs. These tends are shown in Fig. 2.

TABLE I: The calculated equilibrium lattice constants $(a / c)$, the relative volume change $(\delta v)$ with respect to the pure AlN, the magnetic moment per magnetic atom $(M)$, and the electronic density of states at the Fermi level $\left(D_{\mathrm{F}}\right)$. The pure AlN phase is presented for comparison.

\begin{tabular}{ccccc}
\hline \hline Name & $a / c(\AA)$ & $\delta v(\%)$ & $M\left(\mu_{B}\right)$ & $D_{\mathrm{F}}$ \\
\hline $\mathrm{Al}_{36} \mathrm{~N}_{36}+V_{\mathrm{N}}$ & $9.65 / 10.29$ & 1.74 & 1.000 & $>0$ \\
\hline $\mathrm{CrAl}_{35} \mathrm{~N}_{36}$ & $9.59 / 10.26$ & 0.21 & 3.000 & $>0$ \\
\hline $\mathrm{CrAl}_{35} \mathrm{~N}_{36}+V_{\mathrm{N} 1}$ & $9.58 / 10.22$ & -0.16 & 4.000 & 0 \\
\hline $\mathrm{CrAl}_{35} \mathrm{~N}_{36}+\mathrm{N}_{\mathrm{T} 1}$ & $9.66 / 10.26$ & 1.28 & 2.000 & 0 \\
\hline $\mathrm{CrAl}_{35} \mathrm{~N}_{36}+\mathrm{N}_{\mathrm{O} 1}$ & $9.61 / 10.29$ & 1.15 & 4.000 & 0 \\
\hline $\mathrm{Al}_{36} \mathrm{~N}_{36}$ & $9.58 / 10.27$ & 0.00 & 0.000 & 0 \\
\hline \hline
\end{tabular}

Our systematic calculations show that a doped $\mathrm{Cr}$

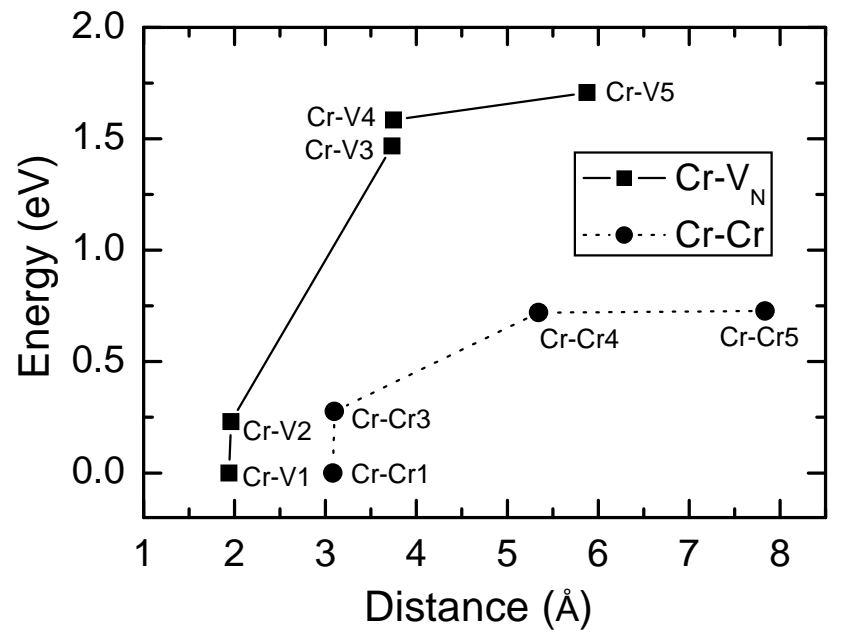

FIG. 2: Relative total energy of the Cr doped AlN with N vacancies for different $\mathrm{Cr}-V_{\mathrm{N}}$ distances (filled squares) and that of $\mathrm{Cr}$ doped $\mathrm{AlN}$ for different $\mathrm{Cr}-\mathrm{Cr}$ distance (filled circles). Here Cr-Crn means the pair of the first $\mathrm{Cr}$ and the second $\mathrm{Cr}$ at the $\mathrm{Al} n$ site, and $\mathrm{Cr}-\mathrm{V} n$ the pair of the first $\mathrm{Cr}$ and the $\mathrm{N}$ vacancy at the $\mathrm{N} n$ site, as are labelled in Fig. 1. The two smallest total energies, corresponding to the nearest $\mathrm{Cr}-V_{\mathrm{N}}$ and $\mathrm{Cr}-\mathrm{Cr}$ pairs, are set to zero.

atom contributes $3 \mu_{B}$ to the total magnetic moment, and a neighboring $\mathrm{N}$-vacancy adds $1 \mu_{B}$ because its electron just below the Fermi level is transferred to the $\mathrm{Cr} d$ state. Actually, an isolate $\mathrm{N}$-vacancy, even without any $\mathrm{Cr}$ doping, has a moment of $1 \mu_{B}$, but $\mathrm{N}$ vacancies are in paramagnetic order because inter-vacancy spin interaction is equivalent to zero within computational error. As for interstitial $\mathrm{N}$ atoms, a tetrahedral $\mathrm{N}$ carries a moment of $-1 \mu_{B}$ and an octahedral $\mathrm{N}$ has $1 \mu_{B}$, but they are too high in total energy to exist in real samples. Two neighboring Cr atoms, such as $\mathrm{Cr}-\mathrm{Cr} 1$ and $\mathrm{Cr}-\mathrm{Cr} 3$, have $6 \mu_{B}$ because their ferromagnetic couplings are favorable in energy. In fact, the ferromagnetic orientation is lower in total energy by $464 \mathrm{meV}$ and $195 \mathrm{meV}$ than the corresponding antiferromagnetic one for $\mathrm{Cr}-\mathrm{Cr} 1$ and $\mathrm{Cr}-\mathrm{Cr} 3$, respectively. The energy difference $464 \mathrm{meV}$ is decreased to $163 \mathrm{meV}$ when one $\mathrm{N}$ vacancy is at the nearest site, and is further reduced to $25 \mathrm{meV}$ when two $\mathrm{N}$ vacancies are in the nearest neighborhood. This trend is consistent with effect of $\mathrm{N}$ vacancy on $\mathrm{Mn}-\mathrm{Mn}$ spin exchange constants 34 . The lattice constants, the relative volume changes, the magnetic moments per supercell of the most stable configurations are summarized in Table I. The most stable is the $\mathrm{N}$ interstitial $\mathrm{N}_{\mathrm{T} 1}\left(\mathrm{~N}_{\mathrm{O} 1}\right)$, the nearest to the $\mathrm{Cr}$ atom, in the $\mathrm{CrAl}_{35} \mathrm{~N}_{36}+\mathrm{N}_{\mathrm{T} 1}\left(\mathrm{CrAl}_{35} \mathrm{~N}_{36}+\mathrm{N}_{\mathrm{O} 1}\right)$, among all the tetrahedral (octahedral) $\mathrm{N}$ interstitials. The corresponding results for the pure AlN are also presented for comparison. The DOS at the Fermi level, $D_{F}$, is zero in all the cases except for the $\mathrm{CrAl}_{35} \mathrm{~N}_{36}$ whose Fermi level is at the $\mathrm{Cr} d$ impurity bands and the $\mathrm{Al}_{36} \mathrm{~N}_{36}+V_{\mathrm{N}}$ whose Fermi level is at the N-vacancy-induced in-gap band.

We further calculate formation energy for all the cases. 
The formation energy of the supercell including $n_{V_{\mathrm{N}}} \mathrm{N}$ vacancies and $n_{\mathrm{Cr}}$ substitutional $\mathrm{Cr}$ atoms is defined as $\underline{41.42}$

$$
\begin{aligned}
E_{f}\left(n_{V_{\mathrm{N}}}, n_{\mathrm{Cr}}\right)= & E\left(n_{V_{\mathrm{N}}}, n_{\mathrm{Cr}}\right)-E(0,0) \\
& +n_{V_{\mathrm{N}}} \mu_{\mathrm{N}}-n_{\mathrm{Cr}} \mu_{\mathrm{Cr}}+n_{\mathrm{Cr}} \mu_{\mathrm{Al}}
\end{aligned}
$$

where $E\left(n_{V_{\mathrm{N}}}, n_{\mathrm{Cr}}\right)$ is the total energy of the supercell, and $\left(\mu_{\mathrm{N}}, \mu_{\mathrm{Cr}}, \mu_{\mathrm{Al}}\right)$ are chemical potentials of $\mathrm{N}, \mathrm{Cr}$, and Al. Using $\mu_{\mathrm{N}}^{\text {gas }}, \mu_{\mathrm{Al}}^{\text {bulk }}$, and $\mu_{\mathrm{Cr}}^{\text {bulk }}$ as the chemical potentials of $\mathrm{N}$ gas, $\mathrm{Cr}$ bulk, and $\mathrm{Al}$ bulk, we have $\mu_{\mathrm{N}}=$ $\mu_{\mathrm{N}}^{\text {gas }}+\bar{\mu}_{\mathrm{N}}, \mu_{\mathrm{Al}}=\mu_{\mathrm{Al}}^{\text {bulk }}+\bar{\mu}_{\mathrm{Al}}$, and $\mu_{\mathrm{Cr}}=\mu_{\mathrm{Cr}}^{\text {bulk }}+\bar{\mu}_{\mathrm{Cr}}$. The thermodynamical stability of AlN requires

$$
\bar{\mu}_{\mathrm{N}}+\bar{\mu}_{\mathrm{Al}}=\bar{\mu}_{\mathrm{AlN}} .
$$

The fact that there is no spontaneous formation of $\mathrm{CrN}$ implies

$$
\bar{\mu}_{\mathrm{N}}+\bar{\mu}_{\mathrm{Cr}} \leq \bar{\mu}_{\mathrm{CrN}} .
$$

We can express the minimal formation energy as

$$
\begin{gathered}
E_{f}^{\min }\left(n_{V_{\mathrm{N}}}, n_{\mathrm{Cr}}\right)=E\left(n_{V_{\mathrm{N}}}, n_{\mathrm{Cr}}\right)-E(0,0) \\
\quad+n_{V_{\mathrm{N}}} \mu_{\mathrm{N}}^{\text {gas }}-n_{\mathrm{Cr}}\left(\mu_{\mathrm{Cr}}^{\mathrm{bulk}}-\mu_{\mathrm{Al}}^{\mathrm{bulk}}\right) \\
\quad+n_{V_{\mathrm{N}}} \bar{\mu}_{\mathrm{N}}^{\min }-n_{\mathrm{Cr}}\left(\bar{\mu}_{\mathrm{Cr}}^{\mathrm{max}}-\bar{\mu}_{\mathrm{Al}}^{\min }\right)
\end{gathered}
$$

where the superscripts max (min) means the maximum (minimum) of the chemical potential. The chemical potentials $\mu_{\mathrm{N}}^{\text {gas }}, \mu_{\mathrm{Al}}^{\text {bulk }}$, and $\mu_{\mathrm{Cr}}^{\text {bulk }}$ can be calculated in terms of total energies of $\mathrm{N}$ gas, Al bulk, and Cr bulk. The maximal and minimal chemical potentials can be obtained in terms of equality (2) and inequality (3) and, therefore, the minimal formation energy can be calculated in terms of Eq. (44) as long as $\bar{\mu}_{\mathrm{CrN}}$ and $\bar{\mu}_{\mathrm{AlN}}$ are known.

TABLE II: The formation energies $(\Delta E)$ of a substitutional $\mathrm{Cr}$ atom and an $\mathrm{N}$ vacancy, and their pairs in AlN. The '+', ' 0 ', and '-' superscripts mean that the extra charge is equivalent to $e, 0$, and $-e$, where $e$ is the absolute value of the electronic charge. The last row corresponds to the case that the distance between $\mathrm{Cr}$ and $V_{\mathrm{N}}$ is the longest within the $3 \times 3 \times 2$ supercell, and the fourth row the case that the distance is the shortest.

\begin{tabular}{cc}
\hline \hline Name & $\Delta E(\mathrm{eV})$ \\
\hline $\mathrm{Al}_{36} \mathrm{~N}_{36}+V_{\mathrm{N} 1}$ & -0.23 \\
\hline $\mathrm{Al}_{36} \mathrm{~N}_{36}+V_{\mathrm{N}}^{+}$ & $0.1^{44}$ \\
\hline $\mathrm{Cr}^{0} \mathrm{Al}_{35} \mathrm{~N}_{36}$ & 0.04 \\
\hline $\mathrm{Cr}^{-} \mathrm{Al}_{35} \mathrm{~N}_{36}+V_{\mathrm{N} 1}^{+}$ & 0.77 \\
\hline $\mathrm{Cr}^{-} \mathrm{Al}_{35} \mathrm{~N}_{36}+V_{\mathrm{N} 5}^{+}$ & 2.47 \\
\hline
\end{tabular}

Our calculated result is $\bar{\mu}_{\mathrm{AlN}}=-3.33 \mathrm{eV}$ for $\mathrm{AlN}$, and we use known data $\bar{\mu}_{\mathrm{CrN}}=-1.30 \mathrm{eV}$ for $\mathrm{CrN}^{43}$. For a single neutral $\mathrm{N}$-vacancy in AlN under poor $\mathrm{N}$ condition, we have $n_{V_{\mathrm{N}}}=1$ and $n_{\mathrm{Cr}}=0$, and thus the formation energy is $-0.23 \mathrm{eV}$, while the formation energy of a single $\mathrm{N}^{+}$vacancy is $0.1 \mathrm{eV}^{\underline{44}}$. For a neutral $\mathrm{Cr}$ atom under rich $\mathrm{N}$ condition, we have $n_{V_{\mathrm{N}}}=0$ and $n_{\mathrm{Cr}}=1$, and thus the formation energy is $0.04 \mathrm{eV}$. This small formation energy
$0.04 \mathrm{eV}$ is reasonable and consistent with experimental high $\mathrm{Cr}$ doping concentrations 16,17,24,29,30. A previous unreasonably high formation energy $3.01 \mathrm{eV}^{45}$ is in contradiction with the experimental high $\mathrm{Cr}$ doping concentrations. Charged $\mathrm{Cr}$ atoms have much higher formation energies. For the nearest $\mathrm{Cr}-V_{\mathrm{N} 1}$ pair, we have $n_{V_{\mathrm{N}}}=1$ and $n_{\mathrm{Cr}}=1$, and thus the formation energy is $0.77 \mathrm{eV}$, while more separated $\mathrm{Cr}-V_{\mathrm{N} 5}$ pairs have higher formation energies. These results are summarized in Table II. Interstitial N atoms, both tetrahedral and octahedral, have very high total energies and formation energies, and therefore are not presented in Table II. This implies that either dilute $\mathrm{N}$ vacancies or dilute $\mathrm{Cr}$ atoms are easy to be realized in AlN because the interactions can be neglected in the dilute doping limit.

\section{ELECTRONIC STRUCTURES}

We present spin-dependent DOS of the $\mathrm{Al}_{36} \mathrm{~N}_{35}$ (including one $\mathrm{N}$ vacancy in the supercell), the $\mathrm{CrAl}_{35} \mathrm{~N}_{36}$ (including one $\mathrm{Cr}$ substitutional at an $\mathrm{Al}$ site), and the $\mathrm{CrAl}_{35} \mathrm{~N}_{35}$ (including one $\mathrm{Cr}+V_{\mathrm{N} 1}$ complex) in Fig. 3 . The valence bands for every spin channel are filled with 108 electrons for the AlN supercell, and in total we have 216 electrons in the valence bands. The absence of one $\mathrm{N}$ atom removes $3 p$ electrons and re-organizes the band structures, as shown in Fig. 3(a). Now there are three electrons in the gap of AlN and the valence band manifold is filled with 210 electrons, 105 for each spin channel. The $\mathrm{N}$ vacancy cannot donate electron to the conduction bands because the Fermi level is too low with respect to the conduction bands. This is different from the Nvacancy of $\mathrm{GaN}^{4 \underline{46}}$. Fig. 3.(b) shows the DOS of the $\mathrm{CrAl}_{35} \mathrm{~N}_{36}$. In this case we have 108 electrons in the valence band manifold for each spin channel and the $\mathrm{Cr} d$ states are in the gap of AlN. The $d$ states are split into a doublet (labelled as 'II') and a triplet (labelled as 'I'). The former is filled with two electrons and the latter by one. The Fermi level is in the bands of the triplet. Hence one $\mathrm{Cr}$ contributes $3 \mu_{B}$ to the moment. In Fig. 3(c) we show the density of states of the $\mathrm{CrAl}_{35} \mathrm{~N}_{35}$. The interaction of the doped $\mathrm{Cr}$ and the $\mathrm{N}$ vacancy makes the filled singlet originating from the $\mathrm{N}$ vacancy move lower in energy and become two spin-split parts: 'a' and 'b', and at the same time makes the $\mathrm{Cr} d$ triplet split into a doublet (also labelled as ' $\mathrm{I}$ ' in Fig. 3(c)) and a singlet. The valence band manifold below the part ' $\mathrm{b}$ ' consists of $105+105$ filled states. As a result, one $\mathrm{Cr}+V_{\mathrm{N}}$ complex contributes $4 \mu_{B}$ to the moment.

We present in Fig. 4 electron density distribution of the N-vacancy-induced filled states, the parts ' $a$ ' and ' $b$ ' indicated in Fig. 3(c), of the $\mathrm{CrAl}_{35} \mathrm{~N}_{35}$. The three planes are defined by three-site series: $\mathrm{Cr}-V_{\mathrm{N}}-\mathrm{Al} 3, \mathrm{Cr}-$ $V_{\mathrm{N}}-\mathrm{Al} 2$, and $\mathrm{Al} 2-V_{\mathrm{N}}-\mathrm{Al} 3$. Here $\mathrm{Cr}, \mathrm{Al} 1, \mathrm{Al} 2, \mathrm{Al} 3$ are the atoms labelled with the same signs in Fig. 1. It can be seen that the charge density distribution at the $\mathrm{N}$ vacancy is about $0.014 e /$ a.u. ${ }^{3}$, where $e$ is the absolute value 


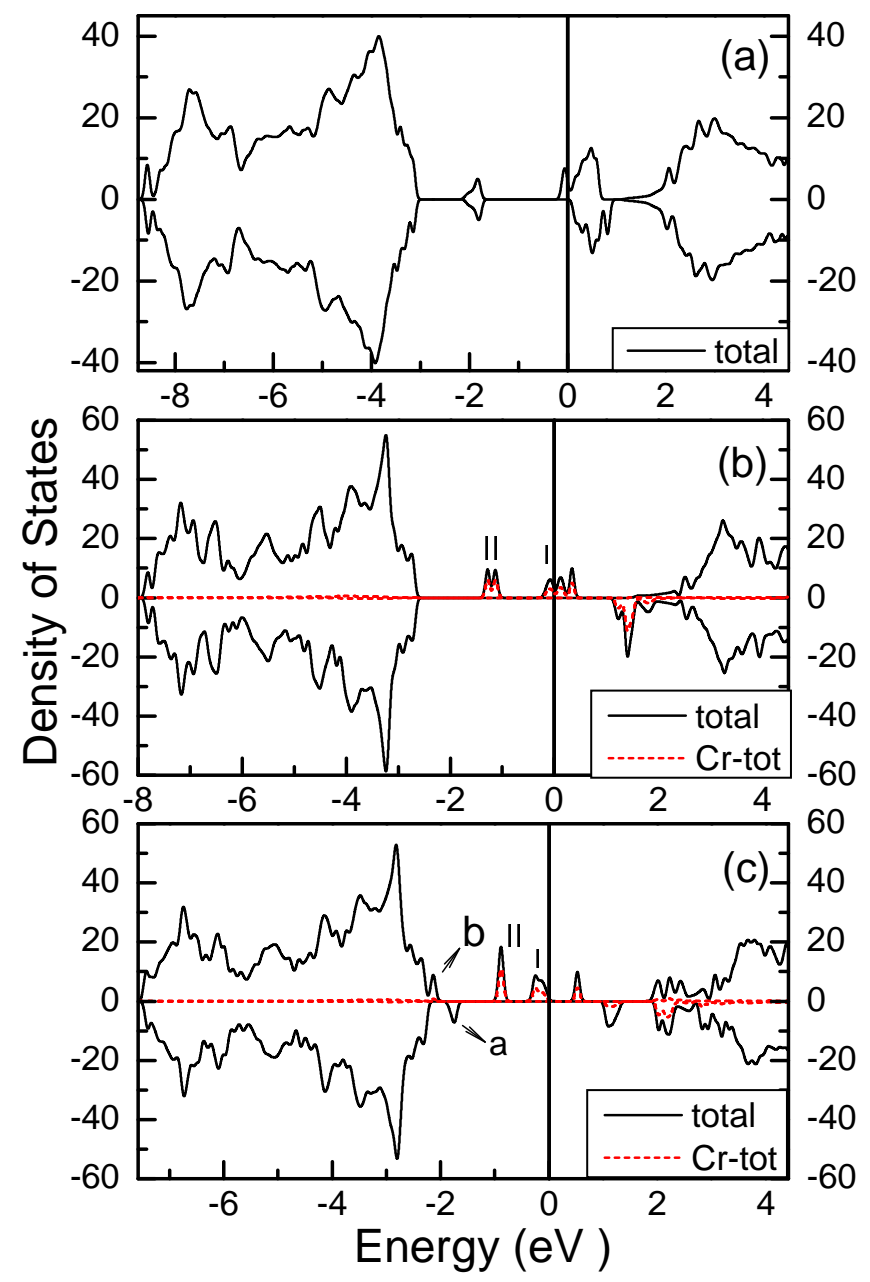

FIG. 3: (color online). Spin-dependent total DOS (black solid lines) and Cr $d$ partial DOS (red or gray dash lines) of the $\mathrm{Al}_{36} \mathrm{~N}_{35}$ (including one $V_{\mathrm{N}}$ ) (a), the $\mathrm{CrAl}_{35} \mathrm{~N}_{36}$ (including one Cr atom) (b), and the $\mathrm{CrAl}_{35} \mathrm{~N}_{35}$ (including one $\mathrm{Cr}+V_{\mathrm{N} 1}$ complex) (c). The upper half for each panel is for majority spin channel and the other for minority spin. For the $\mathrm{Al}_{36} \mathrm{~N}_{35}$, the filled part just below the Fermi level contains on electron, and thus one $V_{\mathrm{N}}$ contributes $1 \mu_{B}$. For the $\mathrm{CrAl}_{35} \mathrm{~N}_{36}$, there is one electron in the small part 'I' and two in the filled part of 'II', and hence the total moment is $3 \mu_{B}$. For the $\mathrm{CrAl}_{35} \mathrm{~N}_{35}$, both 'I' and 'II' are filled with two electrons, and the total moment is $4 \mu_{B}$. The small parts ' $a$ ' and 'b', both filled with one electron, are due to $V_{\mathrm{N}}$.

of the electronic charge. The total charge within the $\mathrm{N}$ vacancy is estimated to be approximately equivalent to 1.1e. The remaining $0.9 e$ charge of the parts ' $\mathrm{a}$ ' and ' $\mathrm{b}$ ' is distributed at the nearest $\mathrm{Cr}$ site $(0.6 e)$ and $\mathrm{N}$ site $(0.3 e)$. This indicates that the $\mathrm{N}$-vacancy state is substantially hybridized with the $\mathrm{Cr} d$ states and substantial charge is transferred to the vacancy from the neighboring $\mathrm{Al}$ (or $\mathrm{Cr}$ ) atoms although there is no $\mathrm{N}$ atom at the site.

We present in Fig. 5 the $\mathrm{Cr} d$ electron density distribution of the $\mathrm{CrAl}_{35} \mathrm{~N}_{36}$ and the $\mathrm{CrAl}_{35} \mathrm{~N}_{35}$. The three planes are the same as those in Fig. 4. For the

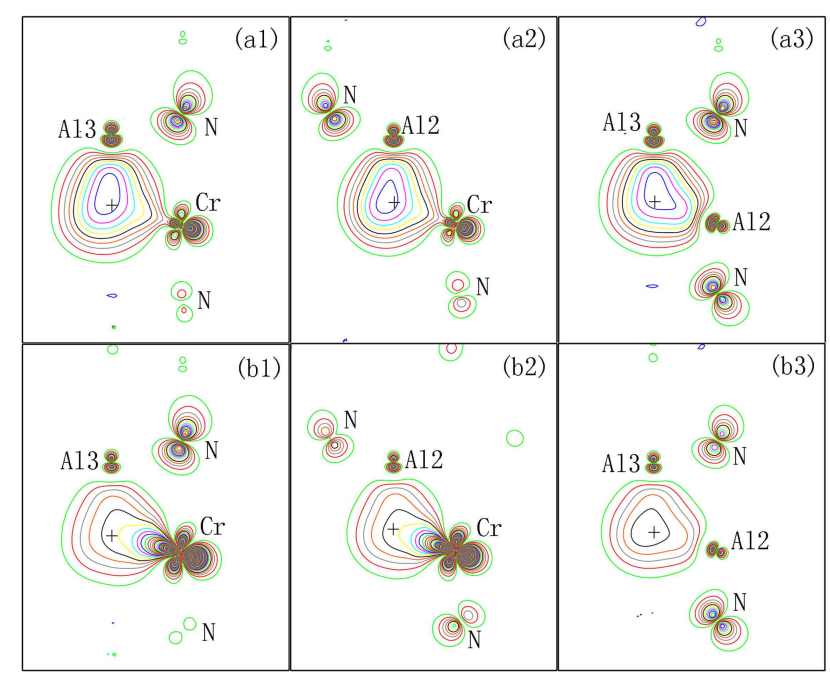

FIG. 4: (color online). Electron density distributions of the ' $a$ ' and ' $b$ ' bands of the $\mathrm{CrAl}_{35} \mathrm{~N}_{35}$, defined in panel (c) of Fig. 3. The a1, a2, and a3 (b1, b2, and b3) show the electron density distributions of the ' $a$ ' (' $b$ ') bands in the three planes defined by $\mathrm{Cr}-V_{\mathrm{N} 1}-\mathrm{Al} 3, \mathrm{Cr}-V_{\mathrm{N} 1}-\mathrm{Al} 2$, and $\mathrm{Al} 2-V_{\mathrm{N} 1}-\mathrm{Al} 3$, where Cr, Al1, Al2, Al3 means the atoms labelled with the same signs in Fig. 1 and the $V_{\mathrm{N} 1}$ is at the $\mathrm{N} 1$ site, labelled as '+', connecting the $\mathrm{Cr}$ atom and three $\mathrm{Al}$ ones. The outmost (green or light gray) line represents $0.001 \mathrm{e} / \mathrm{a} . \mathrm{u}^{3}{ }^{3}$. The density contour increment is $0.001 \mathrm{e} / \mathrm{a} . \mathrm{u}^{3}$.

$\mathrm{CrAl}_{35} \mathrm{~N}_{36}$, it is estimated approximately that $80 \%$ of the 'II' charge is around the $\mathrm{Cr}$ atom and $70 \%$ of the ' $\mathrm{I}$ ' charge around the $\mathrm{Cr}$, and the $\mathrm{Cr}$ electronic configuration is $d^{3}$. For the $\mathrm{CrAl}_{35} \mathrm{~N}_{35}$, approximately $90 \%$ of the 'II' charge is around the $\mathrm{Cr}$ atom and $85 \%$ of the 'I' charge around the $\mathrm{Cr}$. The remaining charge is around the nearest $\mathrm{N}$ sites. Therefore, we can conclude that $\mathrm{Cr}$ electronic configuration in a $\mathrm{Cr}+V_{\mathrm{N}}$ complex is $d^{4}$, not $d^{3}$. The extra electron comes from the $\mathrm{N}$ vacancy. Thus the valent state of the $\mathrm{Cr}+V_{\mathrm{N}}$ complex is $\mathrm{Cr}^{-}+V_{\mathrm{N}}^{+}$.

\section{FERROMAGNETISM CORRELATED WITH N DEFECTS IN REAL SAMPLES}

Because the minimal formation energy for forming a neutral $\mathrm{N}$ vacancy in $\mathrm{AlN}$ is $-0.23 \mathrm{eV}$, a small concentration of $\mathrm{N}$ vacancies can be formed spontaneously in AlN under poor $\mathrm{N}$ condition. An $\mathrm{N}$ vacancy contributes $1 \mu_{B}$ to the moment, but our calculations show that spin interaction between $\mathrm{N}$-vacancy spins is estimated to be zero within computational error. Hence $\mathrm{N}$ vacancies alone cannot form ferromagnetism. Because the minimal formation energy for forming a substitutional $\mathrm{Cr}$ in AlN is $0.04 \mathrm{eV}$, dilute $\mathrm{Cr}$ doping alone is easy to be realized in AlN at room temperature under N-rich condition. High concentrations of $\mathrm{Cr}$ atoms have been doped into AlN 16,17,24,29,30, although the Cr formation energy may be larger when $\mathrm{Cr}-\mathrm{Cr}$ interaction cannot be neglected. 


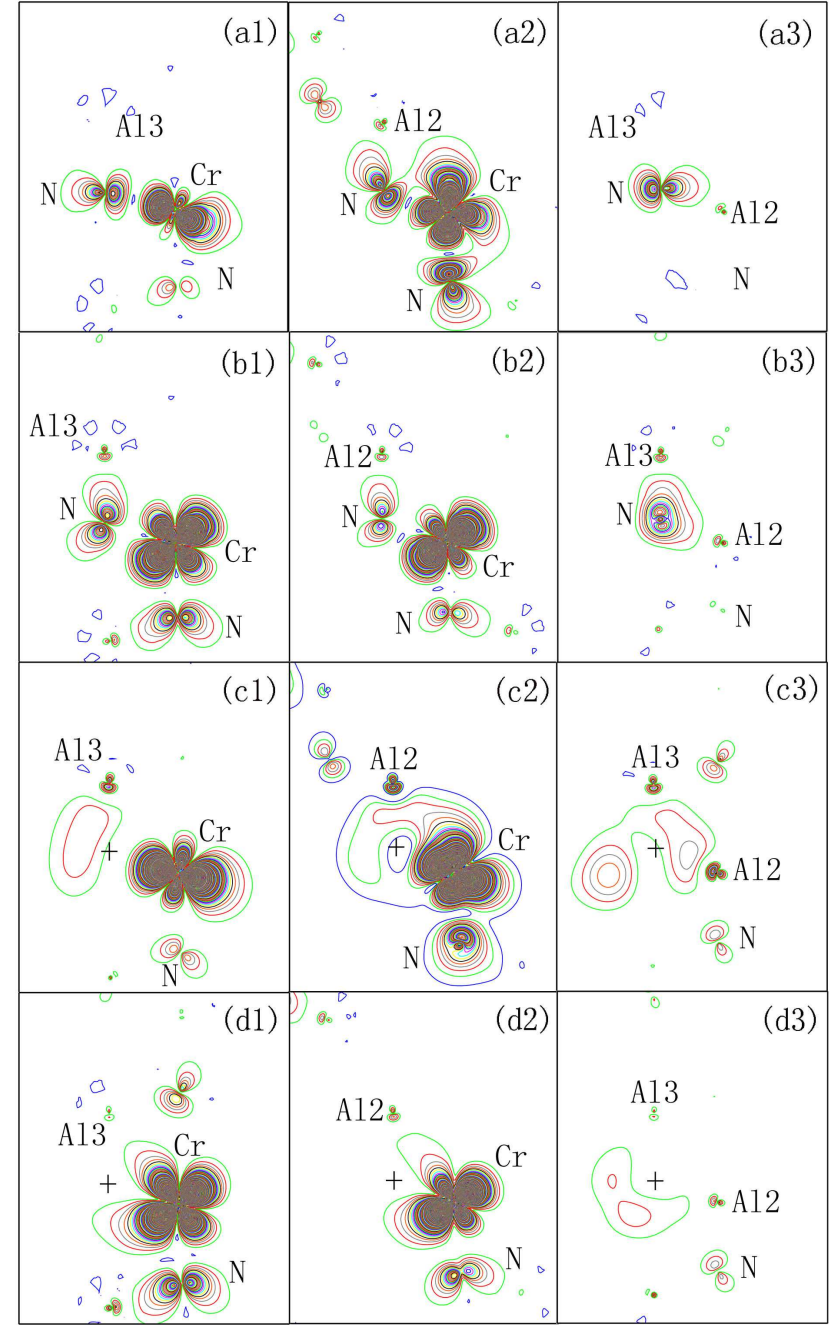

FIG. 5: (color online). Electron density distributions of the parts 'I' (a1, a2, a3) and 'II' (b1, b2, b3) of the $\mathrm{CrAl}_{35} \mathrm{~N}_{36}$, defined in panel (b) of Fig. 3, and those of the parts ' $\mathrm{T}$ ' (c1, $\mathrm{c} 2, \mathrm{c} 3)$ and 'II' (d1, d2, d3) of the $\mathrm{CrAl}_{35} \mathrm{~N}_{35}$, defined in panel (c) of Fig. 3, where the three planes are the same as those in Fig. 4. It should be pointed out that the part 'I' of the $\mathrm{CrAl}_{35} \mathrm{~N}_{35}$ consists of two electrons, but that of the other consists of one electron only. The outmost (green or light gray) line represents $0.001 \mathrm{e} / \mathrm{a} . \mathrm{u}^{3}{ }^{3}$. The density contour increment is $0.001 \mathrm{e} / \mathrm{a} . \mathrm{u} .^{3}$.

This implies that there is no barrier of formation energy in the dilute doping limit. The $\mathrm{Cr}$ doped AlN can be ferromagnetic through some mechanism based on hybridization between local Cr- $d$ states and extended N-p states $^{26,27,28}$.

The minimal formation energy for forming a $\mathrm{Cr}+V_{\mathrm{N}}$ complex is $0.77 \mathrm{eV}$. As a result, the concentration of such complex is at most $2.3 \times 10^{-6}$ per $\mathrm{Al}$ site when the synthesis temperature is at $600 \mathrm{~K}$, becomes $10^{-3}$ at $1140 \mathrm{~K}$, and reaches to $1 \%$ at $1700 \mathrm{~K}$. Each of the $\mathrm{Cr}+V_{\mathrm{N}}$ complexes contributes $4 \mu_{B}$ to the total moment. It should be pointed out that the maximal equilibrium concentra- tion of such complexes at $1000 \mathrm{~K}$ is lower at least by two orders of magnitude than the $\mathrm{Cr}$ concentrations of MBE-grown samples (nominally $2 \sim 7 \%$ )18,19,21,22 and other ones (nominally $3 \sim 35 \%) \underline{16,17,24,29,30}$ of $(\mathrm{Al}, \mathrm{Cr}) \mathrm{N}$ diluted magnetic semiconductors. Thus the synthesis of real samples is not through growth of the $\mathrm{Cr}+V_{\mathrm{N}}$ complexes.

(a)

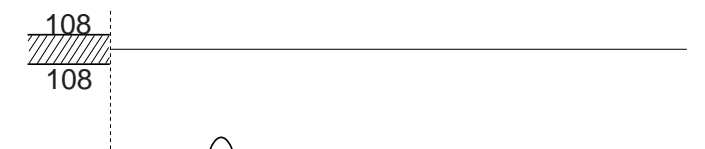

(b)

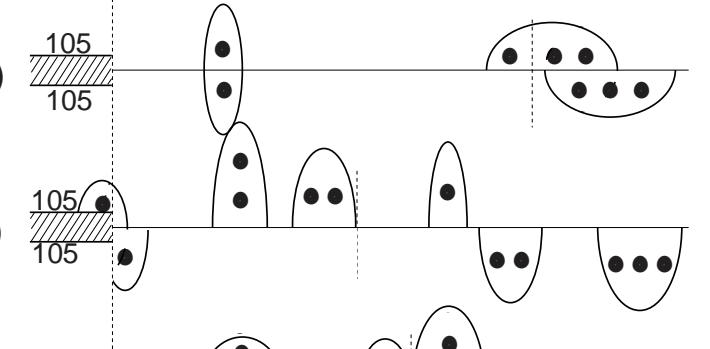

(d)

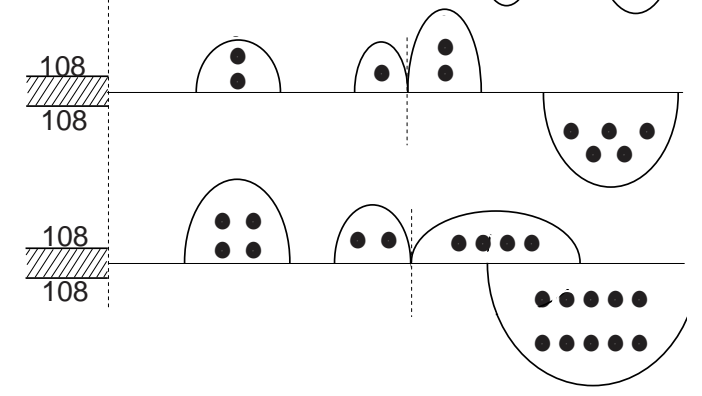

FIG. 6: Schematic DOS of the $\mathrm{Al}_{36} \mathrm{~N}_{36}$ (a), the $\mathrm{Al}_{36} \mathrm{~N}_{35}$ (including one $V_{\mathrm{N}}$ ) (b), the $\mathrm{CrAl}_{35} \mathrm{~N}_{35}$ (including one $\mathrm{Cr}+V_{\mathrm{N} 1}$ complex) (c), the $\mathrm{CrAl}_{35} \mathrm{~N}_{36}$ (including one substitutional $\mathrm{Cr}$ atom) (d), and the $\mathrm{Cr}_{2} \mathrm{Al}_{34} \mathrm{~N}_{36}$ (including two nearest substitutional Cr atoms) (e). The two numbers for the shadow region denote the numbers of the majority-spin $p$ valence bands and minority-spin ones. A solid dot means a filled electron in the bands.

It is reasonable to suppose that there coexist $\mathrm{N}$ vacancies, $\mathrm{Cr}$ atoms, and $\mathrm{Cr}+V_{\mathrm{N}}$ complexes in real samples and the fractional concentrations are dependent on synthesis conditions $\frac{18,19}{19}$. Because of $\mathrm{N}$ pressure fluctuation during synthesis, a substitutional $\mathrm{Cr}$ and an $\mathrm{N}$ vacancy can be formed easily in different regions. This implies that some N-vacancy domains and some $\mathrm{Cr}$-doping domains can be formed simultaneously in separated different regions. The former are paramagnetic and the latter are ferromagnetic, but these domains are subject to thermodynamical equilibrium of electrons in real samples. We present the schematic DOS and the corresponding Fermi levels of the $\mathrm{Al}_{36} \mathrm{~N}_{36}$, the $\mathrm{Al}_{36} \mathrm{~N}_{35}$ (including one $\mathrm{N}$ vacancy in the supercell), the $\mathrm{CrAl}_{35} \mathrm{~N}_{35}$ (including one $\mathrm{Cr}+V_{\mathrm{N} 1}$ complex), the $\mathrm{CrAl}_{35} \mathrm{~N}_{36}$ (including one $\mathrm{Cr}$ substitutional at an $\mathrm{Al}$ site), and the $\mathrm{Cr}_{2} \mathrm{Al}_{34} \mathrm{~N}_{36}$ (including $\mathrm{Cr}$ and $\mathrm{Cr} 1$ as defined in Fig. 1) in Fig. $6(\mathrm{a} \sim \mathrm{e})$. The Fermi level of the $\mathrm{CrAl}_{35} \mathrm{~N}_{36}$ is consistent with that of the $\mathrm{Cr}_{2} \mathrm{Al}_{34} \mathrm{~N}_{36}$. Because the Fermi level of the $\mathrm{N}$ vacancy is substantially higher than that of the $\mathrm{Cr}$ atom, 
the electron of the $\mathrm{N}$ vacancy must transfer to the $\mathrm{Cr}$ site and this electron transfer results in pairs of $V_{\mathrm{N}}^{+}$and $\mathrm{Cr}^{-}$. The Coulomb attraction between $V_{\mathrm{N}}^{+}$and $\mathrm{Cr}^{-}$will make them tend to move together and form the neutral $\mathrm{Cr}+V_{\mathrm{N}}$ complex. Both of the Fermi levels of the $V_{\mathrm{N}}^{+}$and the neutral $\mathrm{Cr}+V_{\mathrm{N}}$ complex are consistent with that of $\mathrm{Cr}$ atom. The electron of the $\mathrm{N}$ vacancy tends to be transferred to the $\mathrm{Cr}$ atom and the neutral $\mathrm{Cr}+V_{\mathrm{N}}$ complex.

The $\mathrm{N}$ vacancy is in favor of the ferromagnetism through forming the $\mathrm{Cr}+V_{\mathrm{N}}$ complex which contributes $4 \mu_{B}$ to the moment and $\mathrm{Cr}+2 V_{\mathrm{N}}$ complex which contributes $5 \mu_{B}$. On the other hand, the $\mathrm{N}$ vacancy can be harmful to the ferromagnetism through destroying the ferromagnetic spin coupling between two nearest substitutional $\mathrm{Cr}$ atoms. We can figure out what will happen in terms of the two characteristic synthesis temperatures, $T_{s 1}$ and $T_{s 2}\left(>T_{s 1}\right)$, for $\mathrm{Cr}_{x} \mathrm{Al}_{1-x} \mathrm{~N}_{1-y}$, where $x$ is $\mathrm{Cr}$ concentration and $y$ is $\mathrm{N}$-vacancy concentration. We suppose that there is no second phase and hence set $y<2 x$ in the following 20 . When $T<T_{s 1}$, neither N-vacancy nor $\mathrm{Cr}$ atom can diffuse. A little of the $\mathrm{N}$-vacancy electrons will transfer to $\mathrm{Cr}$ domains but further electron transferring will be blocked by the built-in electric field formed by the transferred electrons. As a result, the main feathers of the domain patterns will remain. The Cr moments are coupled by thermally-activated variablerange hopping of electrons $\frac{24}{4}$, and thus the $\mathrm{N}$-vacancy enhances the ferromagnetism 22.30 . When $T_{s 1}<T<T_{s 2}$, $\mathrm{N}$-vacancy can diffuse, but $\mathrm{Cr}$ atom cannot. All the Nvacancies and their electrons will transfer to $\mathrm{Cr}$ domains, and many $\mathrm{Cr}+V_{\mathrm{N}}$ and $\mathrm{Cr}+2 V_{\mathrm{N}}$ complexes are formed. In this case, the coupling of moments is the same, but the conductivity becomes weaker because an additional barrier must be overcome for the electron to hop $\underline{24}$, and thus the enhancement decreases with increasing $y$. When $T>T_{s 2}$, both $\mathrm{N}$-vacancy and $\mathrm{Cr}$ atom can diffuse. $\mathrm{Cr}$ atoms tend to form clusters $\$ 5$ (similar to $\mathrm{Cr}$ - and $\mathrm{Mn}$ doped $\mathrm{GaN}^{47,48,49}$ ) and the $\mathrm{N}$ vacancies tend to change the inter-Cr ferromagnetic coupling into antiferromagnetic coupling. In this temperature range, the $\mathrm{N}$-vacancy is harmful to the ferromagnetism. However, The N vacancy can be made to enhance the ferromagnetism by keeping the synthesis temperature below $T_{s 2}$ so that $\mathrm{Cr}$ clusters are unlikely. This is in good agreement with experimental observation that $\mathrm{N}$ vacancies enhance the ferromagnetism in samples made at low temperature 22,30 .

\section{CONCLUSION}

We have investigated nitrogen defects and their effects on the ferromagnetism of $\mathrm{Cr}$-doped AlN with $\mathrm{N}$ vacancies using the full-potential density-functional-theory method and supercell approach. We investigate the structural and electronic properties of the $V_{\mathrm{N}}$, single $\mathrm{Cr}$ atom, $\mathrm{Cr}$ $\mathrm{Cr}$ atom pairs, $\mathrm{Cr}-V_{\mathrm{N}}$ pairs, and so on. In each case, the most stable structure is obtained by comparing different atomic configurations optimized in terms of the total energy and the force on every atom, and then is used to calculate the defect formation energy and study the electronic structures. Without $\mathrm{N}$ vacancy, two $\mathrm{Cr}$ atoms have the lowest energy when they stay at the nearest $\mathrm{Al}$ sites and their moments orient in parallel. An $\mathrm{N}$ vacancy and a $\mathrm{Cr}$ atom tend to form a stable complex of $\mathrm{Cr}^{-}$and $V_{\mathrm{N}}^{+}$and the moment of $4 \mu_{B}$ origins from the $\mathrm{Cr} d^{4}$ electrons. Our calculated formation energies indicate that $V_{\mathrm{N}}$ regions can be formed spontaneously under N-poor condition or high enough concentrations of $\mathrm{Cr}$ atoms can be formed under N-rich condition, but only tiny concentrations of $\mathrm{Cr}+V_{\mathrm{N}}$ complexes can be realized under usual experimental conditions. Both of the $\mathrm{N}$ vacancy and $\mathrm{Cr}$ atom create states in the semiconductor gap of AlN and the highest filled state of the $\mathrm{N}$ vacancy is higher than the filled $\mathrm{Cr}$ states. The $V_{\mathrm{N}}$ enhances the ferromagnetism by giving one electron to $\mathrm{Cr}$ ion to add $1 \mu_{B}$ to the $\mathrm{Cr}$ moment, but reduces the ferromagnetic exchange constants between the spins in the nearest $\mathrm{Cr}-\mathrm{Cr}$ pairs.

The formation energy results imply that real Crdoped samples with $\mathrm{N}$ vacancies are formed by forming Cr-doped regions and some separated $V_{\mathrm{N}}$ regions and through subsequent atomic relaxation during annealing. The $V_{\mathrm{N}}$ enhancement of the ferromagnetism is in good agreement with experimental observation that low nitrogen pressure enhances the ferromagnetism of $\mathrm{Al}_{1-x} \mathrm{Cr}_{x} \mathrm{~N}_{1-y}$ when the synthesis temperature is low so that $\mathrm{Cr}$ atoms cannot diffuse ${ }^{22,30}$. On the other hand, too many $\mathrm{N}$ vacancies are harmful to the ferromagnetism when the synthesis temperature is high enough to make $\mathrm{Cr}$ atoms diffuse and tend to become clusters of secondary phases in which $\mathrm{Cr}-\mathrm{Cr}$ spin interactions mainly are antiferromagnetic $21,22,29,30,31$. However, we can make $\mathrm{N}$-vacancies enhance the ferromagnetism by keeping the synthesis temperature low enough to avoid $\mathrm{Cr}$ diffusion. All these first-principles results are useful to exploring high-performance Cr-doped AlN DMS materials and understanding their high temperature ferromagnetism.

\section{Acknowledgments}

This work is supported by Nature Science Foundation of China (Grant Nos. 10874232, 10774180, 90406010, and 60621091), by the Chinese Academy of Sciences (Grant No. KJCX2.YW.W09-5), and by Chinese Department of Science and Technology (Grant No. 2005CB623602).
* Present address: Institute of Semiconductors, Chinese Academy of Sciences, 100083 Beijing, China $\dagger$ Corresponding author. Email: bgliu@mail.iphy.ac.cn 
1 S. A. Wolf, D. D. Awschalom, R. A. Buhrman, J. M. Daughton, S. von Molnar, M. L. Roukes, A. Y. Chtchelkanova, and D. M. Treger, Science 294, 1488 (2001).

2 T. Dietl, H. Ohno, F. Matsukura, J. Cibert, and D. Ferrand, Science 287, 1019 (2000).

3 S. Sanvito, P. Ordejon, and N. A. Hill, Phys. Rev. B 63, 165206 (2001).

4 T. Jungwirth, J. Sinova, J. Masek, J. Kucera, and A. H. MacDonald, Rev. Mod. Phys. 78, 809 (2006), and references therein.

5 J. M. D. Coey, Current Opinion in Solid State and Mater. Sci. 10, 83 (2006).

${ }^{6}$ K. Ueda, H. Tabata, and T. Kawai, Appl. Phys. Lett. 79, 988 (2001).

7 S.-J. Han, T.-H. Jang, Y. B. Kim, B.-G. Park, J.-H. Park, and Y. H. Jeong, Appl. Phys. Lett. 83, 920 (2003).

8 M. H. F. Sluiter, Y. Kawazoe, P. Sharma, A. Inoue, A. R. Raju, C. Rout, and U. V. Waghmare, Phys. Rev. Lett. 94, 187204 (2005).

9 Y. Matsumoto, M. Murakami, T. Shono, T. Hasegawa, T. Fukumura, M. Kawasaki, P. Ahmet, T. Chikyow, S. Koshihara, and H. Koinuma, Science 291, 854 (2001).

10 S. R. Shinde, S. B. Ogale, S. Das Sarma, J. R. Simpson, H. D. Drew, S. E. Lofland, C. Lanci, J. P. Buban, N. D. Browning, V. N. Kulkarni, J. Higgins, R. P. Sharma, R. L. Greene, and T. Venkatesan, Phys. Rev B 67, 115211 (2003).

11 S. B. Ogale, R. J. Choudhary, J. P. Buban, S. E. Lofland, S. R. Shinde, S. N. Kale, V. N. Kulkarni, J. Higgins, C. Lanci, J. R. Simpson, N. D. Browning, S. Das Sarma, H. D. Drew, R. L. Greene, and T. Venkatesan, Phys. Rev. Lett. 91, 077205 (2003).

12 J. M. D. Coey, A. P. Douvalis, C. B. Fitzgerald, and M. Venkatesan, Appl. Phys. Lett. 84, 1332 (2004).

13 J. Philip, A. Punnoose, B. I. Kim, K. M. Reddy, S. Layne, J. O. Holmes, B. Satpati, P. R. Leclair, T. S. Santos, and J. S. Moodera, Nat. Meter. 5, 298 (2006).

14 J. M. D. Coey, M. Venkatesan, and C. B. Fitzgerald, Nat. Meter. 4, 173 (2005).

15 S. Sonoda, S. Shimizu, T. Sasaki, Y. Yamamoto, and H. Hori, J. Cryst. Growth 237, 1358 (2002).

16 S. Y. Wu, H. X. Liu, L. Gu, R. K. Singh, L. Budd, M. van Schilfgaarde, M. R. McCartney, D. J. Smith, and N. Newman, Appl. Phys. Lett. 82, 3047 (2003).

17 D. Kumar, J. Antifakos, M. G. Blamire, and Z. H. Barber, Appl. Phys. Lett. 84, 5004 (2004).

18 H. X. Liu, S. Y. Wu, R. K. Singh, L. Gu, D. J. Smith, N. Newman, N. R. Dilley, L. Montes, and M. B. Simmonds, Appl. Phys. Lett. 85, 4076 (2004).

19 L. Gu, S. Y. Wu, H. X. Liu, R. K. Singh, N. Newman, and D. J. Smith, J. Magn. Magn. Mater. 290-291, 1395 (2005).

20 R. M. Frazier, J. Stapleton, G. T. Thaler, C. R. Abernathy, S. J. Pearton, R. Rairigh, J. Kelly, A. F. Hebard, M. L. Nakarmi, K. B. Nam, J. Y. Lin, H. X. Jiang, J. M. Zavada, and R. G. Wilson, J. Appl. Phys. 94, 1592 (2003).

21 A. Y. Polyakov, N. B. Smirnov, A. V. Govorkov, R. M. Frazier, J. Y. Liefer, G. T. Thaler, C. R. Abernathy, S. J. Pearton, and J. M. Zavada, Appl. Phys. Lett. 85, 4067
(2004).

22 R. M. Frazier, G. T. Thaler, J. P. Leifer, J. K. Hite, B. P. Gila, C. R. Abernathy, and S. J. Pearton, Appl. Phys. Lett. 86, 052101 (2005).

23 A. Fara, F. Bernardini, and V. Fiorentini, J. Appl. Phys. 85, 2001 (1999).

24 S. G. Yang, A. B. Pakhomov, S. T. Hung, and C. Y. Wong, Appl. Phys. Lett. 81, 2418 (2002).

25 S. Y. Wu and N. Newman, Appl. Phys. Lett. 89, 142105 (2006).

26 B. Sanyal, O. Bengone, and S. Mirbt, Phys. Rev. B 68, 205210 (2003).

27 M. Wierzbowska, D. Sanchez-Portal, and S. Sanvito, Phys. Rev. B 70, 235209 (2004).

${ }^{28}$ L.-J. Shi and B.-G. Liu, Phys. Rev. B 76, 115201 (2007).

29 J. Zhang, S. H. Liou, and D. J. Sellmyer, J. Phys.: Condens. Matter. 17, 3137 (2005).

30 J. Zhang, X. Z. Li, B. Xu, and D. J. Sellmyera, Appl. Phys. Lett. 86, 212504 (2005).

31 Y. Endo, T. Sato, A. Takita, Y. Kawamura, and M. Yamamoto, IEEE Transactions On Magnetics 41, 2718 (2005).

32 A. J. Behan, A. Mokhtari, H. J. Blythe, D. Score, X-H. $\mathrm{Xu}$, J. R. Neal, A. M. Fox, and G. A. Gehring, Phys. Rev. Lett. 100, 047206 (2008).

33 S. Yoshii, S. Sonoda, T. Yamamoto, T. Kashiwagi, M. Hagiwara, Y. Yamamoto, Y. Akasaka, K. Kindo, and H. Hori, EuroPhys. Lett. 78, 37006 (2007).

34 P. Larson and S. Satpathy, Phys. Rev. B 76, 245205 (2007).

35 P. Hohenberg and W. Kohn, Phys. Rev. 136, B864 (1964); W. Kohn and L. J. Sham, Phys. Rev. 140, A1133 (1965).

36 P. Blaha, K. Schwarz, P. Sorantin, and S. B. Trickey, Comput. Phys. Commun. 59, 399 (1990).

37 J. P. Perdew, K. Burke, and M. Ernzerhof, Phys. Rev. Lett. 77, 3865 (1996).

38 J. P. Perdew and Y. Wang, Phys. Rev. B 45, 13244 (1992).

39 Ph. Mavropoulos, K. Sato, R. Zeller, P. H. Dederichs, V. Popescu, and H. Ebert, Phys. Rev. B 69, 054424 (2004).

40 Q. Wang, A. K. Kandalam, Q. Sun, and P. Jena, Phys. Rev. B 73, 115411 (2006).

41 S. B. Zhang and J. E. Northrup, Phys. Rev. Lett. 67, 2339 (1991).

42 S. B. Zhang and S.-H. Wei, Phys. Rev. Lett. 86, 1789 (2001)

43 L. Bellard, C. Fauquet-Ben Ammar, J.-M. Themlin, and A. Cros J. Appl. Phys. 83, 7640 (1998).

44 T. Mattila and R. M. Nieminen, Phys. Rev. B 54, 16676 (1996).

45 X. Y. Cui, D. Fernandez-Hevia, B. Delley, A. J. Freeman, and C. Stampfl, J. Appl. Phys. 101, 103917 (2007).

46 M. G. Ganchenkova and R. M. Nieminen, Phys. Rev. Lett. 96, 196402 (2006).

47 T. Hynninen, H. Raebiger, and J. von Boehm, Phys. Rev. B 75, 125208 (2007).

${ }^{48}$ X. Y. Cui, J. E. Medvedeva, B. Delley, A. J. Freeman, and C. Stampfl, Phys. Rev. B 75, 155205 (2007).

49 X. Y. Cui, B. Delley, A. J. Freeman, and C. Stampfl, Phys. Rev. B 76, 045201 (2007). 